\title{
Evolution of Spacetime arises due to the departure from Holographic Equipartition in all Lanczos-Lovelock Theories of Gravity
}

\author{
Sumanta Chakraborty ${ }^{*}$ and T. Padmanabhan ${ }^{\dagger}$ \\ IUCAA, Post Bag 4, Ganeshkhind, Pune University Campus, Pune 411 00\%, India
}

(Dated: October 20, 2018)

\begin{abstract}
In the case of general relativity one can interpret the Noether charge in any bulk region as the heat content $T S$ of its boundary surface. Further, the time evolution of spacetime metric in Einstein's theory arises due to the difference $\left(N_{\text {sur }}-N_{\text {bulk }}\right)$ of suitably defined surface and bulk degrees of freedom. We show that this thermodynamic interpretation generalizes in a natural fashion to all Lanczos-Lovelock models of gravity. The Noether charge, related to time evolution vector field, in a bulk region of space is equal to the heat content $T S$ of the boundary surface with the temperature $T$ defined using local Rindler observers and $S$ being the Wald entropy. Using the Wald entropy to define the surface degrees of freedom $N_{\text {sur }}$ and Komar energy density to define the bulk degrees of freedom $N_{\text {bulk }}$, we can also show that the time evolution of the geometry is sourced by $\left(N_{\text {sur }}-N_{\text {bulk }}\right)$. When it is possible to choose the foliation of spacetime such that metric is independent of time, the above dynamical equation yields the holographic equipartition for Lanczos-Lovelock gravity with $N_{\text {sur }}=N_{\text {bulk }}$. The implications are discussed.
\end{abstract}

\section{INTRODUCTION}

A surprising connection between gravity and thermodynamics was first demonstrated in the context of black hole mechanics by the fact that one can associate an entropy $[1,2]$ and temperature [3, 4], with the black holes. It was soon realised that similar connection exists in the case of several other horizons $[5,6]$ and that the ideas have a far greater domain of applicability $[7,8]$.

Further work in the last decade suggests that these results could be just the tip of the iceberg. It appears that (i) gravitational field equations themselves may have only the status similar to equations in other emergent phenomenon like in kinetic theory of gases or fluid mechanics [9-12] and (ii) this emergent interpretation is applicable to theories more general than Einstein gravity and has a universal nature. Some of the results which lend support to this paradigm are the following:

- The gravitational field equations reduce to thermodynamic identities on horizons for a wide class of gravity theories more general than Einstein gravity [13-18].

- The action describing gravity can be separated into a bulk term and a surface term with a specific ('holographic') relation between them, not only in Einstein gravity but also in more general class of theories [19-22]. In fact the action functional in all Lanczos-Lovelock gravity can be given a thermodynamic interpretation [22-25].

- Gravitational field equations in all Lanczos-Lovelock models can be obtained from thermodynamic extremum principles $[26,27]$ involving the heat density of null surfaces in the spacetime.

- Gravitational field equations reduce to Navier-Stokes equations of fluid dynamics in arbitrary spacetime projected on a null surface generalizing previous results on black hole spacetime [28-30].

More recently [31] these ideas have been taken significantly further in the context of general relativity. One of us (TP) demonstrated that, in the context of general relativity, the following results hold: (a) The total Noether charge in a 3 -volume $\mathcal{R}$, related to the time evolution vector field, can be interpreted as the heat content of the boundary $\partial \mathcal{R}$ of the volume. This provides yet another holographic result connecting the bulk and boundary variables. (b) The time evolution of the spacetime itself can be described in an elegant manner by the equation:

$$
\int_{\mathcal{R}} \frac{d^{3} x}{8 \pi} h_{a b} £_{\xi} p^{a b}=\epsilon \frac{1}{2} k_{B} T_{\text {avg }}\left(N_{\text {bulk }}-N_{\text {sur }}\right)
$$

\footnotetext{
*sumanta@iucaa.ernet.in; sumantac.physics@gmail.com

† paddy@iucaa.ernet.in
} 
where $h_{a b}$ is the induced metric on the $t=$ constant surface, the $p^{a b}$ is its conjugate momentum, $\xi^{a}=N u^{a}$ is the time evolution vector corresponding to observers with four-velocity $u_{a}=-N \nabla_{a} t$ that is the normal to the $t=$ constant surface. The $N_{\text {sur }}$ and $N_{\text {bulk }}$ are the degrees of freedom in the surface and bulk of a 3-dimensional region $\mathcal{R}$ and $T_{\text {avg }}$ is the average Davies-Unruh temperature of the boundary. (The parameter $\epsilon= \pm 1$ ensures that the $N_{\text {bulk }}$ is positive even when Komar energy turns negative.) This equation shows that the rate of change of gravitational momentum is driven by the departure from holographic equipartition, measured by $\left(N_{\text {bulk }}-N_{\text {sur }}\right)$. The metric will be time independent in the chosen foliation if $N_{\text {sur }}=N_{\text {bulk }}$ which can happen for all static geometries. The validity of Eq. (1) for all observers (i.e., foliations) implies the validity of Einstein's equations. In short, deviation from holographic equipartition leads to the time evolution of the metric.

In the past, virtually every result indicating the emergent nature of gravity in the context of general relativity could be generalized to all Lanczos-Lovelock models of gravity. It is therefore worth investigating whether the above description can be generalized to Lanczos-Lovelock models. This is very important because the expression for horizon entropy in general relativity is rather trivial and is just a quarter of horizon area. In Lanczos-Lovelock models, the corresponding expression is much more complex which, in turn, modifies the expression for $N_{\text {sur }}$. It is, therefore, not clear a priori whether our results interpretation of Noether charge and Eq. (1) — will generalize to Lanczos-Lovelock models. We will show here that, these results indeed possess a natural generalization to Lanczos-Lovelock gravity as well.

The rest of the paper is organised as follows: In Sec. II we review the know results for Einstein gravity and clarify some technical points. (In particular, in Sec II C we give some explicit examples to illustrate what happens when the same spacetime admits both static and nonstatic folications.) In Sec. III we generalise all these results to Lanczos-Lovelock models of gravity. Sec. III A provides a brief introduction to Lanczos-Lovelock models and set up the notation etc. In Sec III B we relate the Noether charge to the surface heat content in the Lanczos-Lovelock models and in Sec III C we derive the evolution equation in terms of surface and bulk degrees of freedom. The last section summarises the conclusions. We work with a mostly positive signature in D dimensional spacetime and use units with $G=\hbar=c=1$.

\section{WARM UP: REVIEW OF THE RESULTS FOR EINSTEIN GRAVITY}

\section{A. The foliation of spacetime}

We start with a spacetime foliated by a series of spacelike hypersurfaces each being determined by the constant value of a scalar field $t(x)$, such that on each hypersurface $t(x)=$ constant. The unit normal to the constant $t(x)$ hypersurface is $u_{a}=-N \nabla_{a} t$, which reduces to $-N \delta_{a}^{0}$ when $t$ is considered as one of the coordinates in this spacetime. For this spacetime foliation we have $g^{00}=-1 / N^{2}$, and $u^{a} u_{a}=-1$. Given such a foliation, we can introduce a time evolution vector $\zeta^{a}$ by the condition $\zeta^{a} \nabla_{a} t=1$, which in the coordinate system with $t$ as a coordinate becomes $\zeta^{a}=\delta_{0}^{a}$. In general, we can readily obtain the following decomposition: $\zeta^{a}=-\left(\zeta^{b} u_{b}\right) u^{a}+N^{a}$, with the property $N^{a} u_{a}=0$ and $N^{a}=h_{b}^{a} \zeta^{b}$, where $h_{b}^{a}=\delta_{b}^{a}+u^{a} u_{b}$ being the projection tensor. This decomposition also introduces another vector

$$
\xi_{a}=N u_{a} \rightarrow-N^{2} \delta_{a}^{0}
$$

where the last result holds in the preferred foliation. If we impose the coordinate condition that $t$ becomes one of the spacetime coordinate and $g_{0 \alpha}=0$ this vector reduces to $\zeta^{a}$. Further, in static spacetimes $\xi^{a}$ turns out to be the time-like Killing vector. It was shown in ref. [31] that this vector plays a crucial role in the thermodynamic interpretation and has a rich structure as far as the Noether current and spacetime dynamics is concerned.

\section{B. Noether Charge and evolution equation in general relativity}

We begin by calculating the Noether charge for the vector field $\xi^{a}$. The Noether current in general

relativity can be written in an elegant manner using a new set of variables $\left(f^{a b}, N_{a b}^{c}\right)$ in terms of which several expressions in general relativity becomes simpler. These variables, defined as:

$$
f^{a b}=\sqrt{-g} g^{a b} ; \quad N_{a b}^{c}=Q_{a e}^{c d} \Gamma_{b d}^{e}+Q_{b e}^{c d} \Gamma_{a d}^{e}
$$


where $2 Q_{c d}^{a b}=\left(\delta_{c}^{a} \delta_{d}^{b}-\delta_{d}^{a} \delta_{c}^{b}\right)$ were earlier used in [32, 33] and their thermodynamic interpretation was provided in [34]. The variation of the Einstein-Hilbert action in terms of these conjugate variables results into:

$$
\begin{aligned}
\delta(\sqrt{-g} R) & =R_{a b} \delta f^{a b}-\partial_{c}\left(f^{a b} \delta N_{a b}^{c}\right) \\
& =\sqrt{-g}\left[G_{a b} \delta g^{a b}-\nabla_{c}\left(g^{i k} \delta N_{i k}^{c}\right)\right]
\end{aligned}
$$

If the above variation results from a Lie variation with respect to some vector field $v_{a}$ then from the above expression a conserved current $J^{a}$ emerges with the property $\nabla_{a} J^{a}=0$. This conserved current is the Noether current and has the following expression:

$$
16 \pi J^{a}(v)=2 R^{a b} v_{b}+g^{i j} £_{v} N_{i j}^{a}
$$

(The factor $16 \pi$ is conventional when we use units with $G=1$; obviously any multiple of $J^{a}$ is conserved.) Given the fact that $\nabla_{a} J^{a}=0$, we can write the Noether current in terms of an antisymmetric second rank tensor $J^{a b}$, the Noether potential as $J^{a}=\nabla_{b} J^{a b}$. This, in the case of general relativity becomes:

$$
16 \pi J^{a b}(v)=\nabla^{a} v^{b}-\nabla^{b} v^{a}
$$

Though in the above discussion the Noether current has been derived using Lie variation it should be stressed that the same result can be obtained using differential geometry without ever using diffeomorphism invariance of the action principle for gravity. This has been shown explicitly in ref. [31] and hence we will not repeat the arguments here.

Next we will calculate the Noether current for the time evolution vector $\xi^{a}$. For the evaluation we shall use a relation between Noether current of two vector fields $q^{a}$ and $v^{a}$ such that $v^{a}=f(x) q^{a}$, for arbitrary function $f(x)$. In App. A 2 [see Eq. (A6)] it is shown that:

$$
16 \pi\left\{q_{a} J^{a}(v)-f(x) q_{a} J^{a}(q)\right\}=\nabla_{b}\left(\left\{q^{a} q^{b}-g^{a b} q^{2}\right\} \nabla_{a} f\right)
$$

The usefulness of this relation can be realized by noting that for $q_{a}=\nabla_{a} \phi$ for some scalar $\phi$ the Noether current vanishes. Thus applying the above result for $u_{a}$ and then for $\xi_{a}$ one can arrive at the following simple relation for Noether current of $\xi_{a}$ as [see App. A 2; Eq. (A9)]:

$$
16 \pi u_{a} J^{a}(\xi)=2 D_{\alpha}\left(N a^{\alpha}\right)
$$

where $a^{i}=u^{j} \nabla_{j} u^{i}$ represents the four acceleration which satisfies the relation $D_{i} a^{i}=\nabla_{i} a^{i}-a^{2}$, with $D_{i}$ representing the surface covariant derivative for the $t=$ constant surface. Then we can integrate Eq. (8) over the $t=$ constant hypersurface with $\sqrt{h} d^{3} x$ being the integration measure and bounded by $N=$ constant surface leading to the total Noether charge contained in the three volume. Then dividing both sides of Eq. (8) by $16 \pi$ we arrive at:

$$
\int_{\mathcal{V}} d^{3} x \sqrt{h} u_{a} J^{a}(\xi)=\int_{\mathcal{V}} \frac{d^{3} x \sqrt{h}}{8 \pi} D_{\alpha}\left(N a^{\alpha}\right)=\int_{\partial \mathcal{V}} \frac{\sqrt{\sigma} d^{2} x}{8 \pi} N r_{\alpha} a^{\alpha}
$$

which holds for any arbitrary region $\mathcal{V}$ of the spacetime, with the bounding region being $N(t, \mathbf{x})=$ constant surface within $t=$ constant hypersurface. This allows us to identify the vector $r_{a}$ to be normal to this $N(t, \mathbf{x})=$ constant hypersurface as: $r_{a}=\epsilon D_{a} N\left(D_{b} N D^{b} N\right)^{-1 / 2}=\epsilon h_{a}^{i} \nabla_{i} N / a$, where the $\epsilon$ factor is introduced to ensure that $r_{a}$ is always the outward pointing normal. (When the acceleration $a_{i}$ is outward pointing $\epsilon=1$; otherwise $\epsilon=-1$ ). Here $a=\sqrt{a_{i} a^{i}}$ is the magnitude of the acceleration. So we can also write the normal $r_{\alpha}$ as: $r_{\alpha}=\epsilon a_{\alpha} / a$, with $a$ representing magnitude of the acceleration. Then we obtain

$$
N r_{\alpha} a^{\alpha}=N \epsilon \frac{a_{\alpha}}{a} a^{\alpha}=\epsilon N a .
$$

The Tolman redshifted Davies-Unruh temperature on the boundary surface $N=$ constant, is $T_{l o c}=$ $N a / 2 \pi$ for observers with four velocity $u_{a}=-N \delta_{a}^{0}$. Locally free falling observers will observe these observers moving normal to the $t=$ constant hypersurface with an acceleration $a$ and as a consequence 
the local vacuum will appear as a thermal state with temperature $T_{l o c}$ to these observers. Using all these results Eq. (9) can be written as:

$$
2 \int_{\mathcal{V}} d^{3} x \sqrt{h} u_{a} J^{a}(\xi)=\epsilon \int_{\partial \mathcal{V}} \frac{\sqrt{\sigma} d^{2} x}{2}\left(\frac{N a}{2 \pi}\right)=\epsilon \int_{\partial \mathcal{V}} \sqrt{\sigma} d^{2} x\left(\frac{1}{2} T_{l o c}\right)
$$

The above result can be interpreted as: twice the Noether charge contained in the $N=$ constant surface is equal to the equipartition energy of the surface. With the interpretation of $\sqrt{\sigma} / 4$ as entropy density the above result also gives:

$$
\int_{\mathcal{V}} d^{3} x \sqrt{h} u_{a} J^{a}(\xi)=\epsilon \int_{\partial \mathcal{V}} \frac{\sqrt{\sigma} d^{2} x}{4} T_{l o c}=\epsilon \int_{\partial \mathcal{V}} d^{2} x T_{l o c} s
$$

which is the heat density of the bounding surface. The interpretation of $\sqrt{\sigma} / 4$ as the entropy density comes naturally when the boundary surface becomes a horizon. Thus, even in the most general (nonstatic) context, the Noether charge of time development vector in the bulk spacetime region has a simple interpretation as the surface heat content.

We will next obtain the dynamics of gravity in terms of bulk and surface degrees of freedom using the Noether current formalism. For this, we again start with Eq. (8) and use Eq. (5) leading to:

$$
u_{a} g^{i j} £_{\xi} N_{i j}^{a}=D_{\alpha}\left(2 N a^{\alpha}\right)-2 N R_{a b} u^{a} u^{b}
$$

Then we integrate the above expression as in the earlier situation over the three dimensional region $\mathcal{R}$ with boundary surface being $N=$ constant within the $t=$ constant surface leading to:

$$
\int_{\mathcal{R}} d^{3} x \sqrt{h} u_{a} g^{i j} £_{\xi} N_{i j}^{a}=\int_{\partial \mathcal{R}} d^{2} x \sqrt{\sigma} r_{\alpha}\left(2 N a^{\alpha}\right)-\int_{\mathcal{R}} d^{3} x \sqrt{h} 2 N R_{a b} u^{a} u^{b}
$$

where we have used $d^{3} x \sqrt{h}$ as the integration measure. Introducing the dynamics through Einstein's equation $R_{a b}=8 \pi\left(T_{a b}-(1 / 2) g_{a b} T\right)=8 \pi \bar{T}_{a b}$ and dividing the whole expression by $8 \pi$ gives:

$$
\int_{\mathcal{R}} \frac{d^{3} x \sqrt{h}}{8 \pi} u_{a} g^{i j} £_{\xi} N_{i j}^{a}=\int_{\partial \mathcal{R}} d^{2} x \sqrt{\sigma} r_{\alpha}\left(\frac{N a^{\alpha}}{4 \pi}\right)-\int_{\mathcal{R}} d^{3} x \sqrt{h} 2 N \bar{T}_{a b} u^{a} u^{b}
$$

Using Eq. (10) and introducing the Komar energy density by the definition $\rho_{\text {Komar }}=2 N \bar{T}_{a b} u^{a} u^{b}$ we obtain:

$$
\frac{1}{8 \pi} \int_{\mathcal{R}} d^{3} x \sqrt{h} u_{a} g^{i j} £_{\xi} N_{i j}^{a}=\epsilon \int_{\partial \mathcal{R}} d^{2} x \sqrt{\sigma}\left(\frac{1}{2} T_{l o c}\right)-\int_{\mathcal{R}} d^{3} x \sqrt{h} \rho_{\text {Komar }}
$$

We define the surface degrees of freedom by:

$$
N_{\text {sur }} \equiv A=\int_{\partial \mathcal{R}} \sqrt{\sigma} d^{2} x
$$

which is always positive. We can define an average temperature over the surface such that

$$
T_{\text {avg }} \equiv \frac{1}{A} \int_{\partial \mathcal{R}} \sqrt{\sigma} d^{2} x T_{l o c}
$$

Finally we introduce the bulk degrees of freedom by the definition:

$$
N_{\text {bulk }}=\frac{\epsilon}{(1 / 2) T_{\text {avg }}} \int d^{3} x \sqrt{h} \rho_{\text {Komar }}
$$

When the bulk region is in equipartition at the temperature $T_{\text {avg }}$ then $N_{\text {bulk }}$ represents the correct number of bulk degrees of freedom. Here also we need the factor $\epsilon$ to ensure that $N_{\text {bulk }}$ is positive definite. We choose $\epsilon=+1$ if the total Komar energy within the volume is positive and $\epsilon=-1$ if the total Komar 
energy in the volume is negative so as to keep $N_{\text {bulk }}$ always positive. With all these definitions Eq. (16) can be written in the following manner: (This corrects a minor typo in ref. [31].)

$$
\frac{1}{8 \pi} \int_{\mathcal{R}} d^{3} x \sqrt{h} u_{a} g^{i j} £_{\xi} N_{i j}^{a}=\frac{\epsilon}{2} T_{a v g}\left(N_{\text {sur }}-N_{\text {bulk }}\right)
$$

Thus for comoving observers in static spacetime we have the holographic equipartition $N_{\text {sur }}=N_{\text {bulk }}$ When the difference $\left(N_{\text {sur }}-N_{\text {bulk }}\right)$ is nonzero for a given foliation, we have departure from holographic equipartition and this leads to the time evolution of the metric, as is evident from the left hand side of Eq. (20). The implications of this result has been discussed extensively in ref. [31].

\section{Aside: Some illustrative examples}

An important aspect of the dynamical evolution equation is the following: The structure of Eq. (20) shows that, while it is covariant, it is foliation dependent through the normal $u_{i}$. For example, even in a static spacetime (which possesses a timelike Killing vector field) the non-static observers will perceive a time-dependence of the metric and hence departure from holographic equipartition (so that both sides of Eq. (20) are nozero), while static observers (with velocities along the Killing direction) will perceive a time-independent metric and holographic equipartition, (with both sides of Eq. (20) being zero). This contrast is most striking when we study two natural class of observers in a static spacetime. The first set are observers with four-velocities along the timelike Killing vector who have a nonzero acceleration. In this foliation the metric components are independent of time and the left hand side of Eq. (20) vanishes leading to holographic equipartition $N_{\text {sur }}=N_{\text {bulk }}$. But we know that any spacetime metric can be expressed in the synchronous frame coordinates with the line element:

$$
d s^{2}=-d \tau^{2}+g_{\alpha \beta} d x^{\alpha} d x^{\beta}
$$

In the synchronous frame the observers at $x^{\alpha}=$ constant are comoving with four velocity: $u_{a}=$ $(-1,0,0,0)$. Obviously, the comoving observer is not accelerating, (i.e, the curves $x^{\alpha}=$ constant are geodesics) and hence the local Davies-Unruh temperature for these observers will vanish. We want to consider Eq. (20) in two such coordinate systems to clarify some of the issues.

Let us begin with the synchronous frame in which $T_{\text {avg }} \rightarrow 0, T_{\text {avg }} N_{\text {sur }} \rightarrow 0$ with $T_{\text {avg }} N_{\text {bulk }}$ remaining finite, so that Eq. (20) reduces to the following form:

$$
\frac{1}{8 \pi} \int_{\mathcal{R}} d^{3} x \sqrt{h} u_{a} g^{i j} £_{\xi} N_{i j}^{a}=-\frac{\epsilon}{2} T_{a v g} N_{b u l k}=-\int_{\mathcal{R}} d^{3} x \sqrt{h} \rho_{\text {Komar }}
$$

The quantity $u_{a} g^{i j} £_{\xi} N_{i j}^{a}$ in an arbitrary synchronous frame is given by:

$$
\begin{aligned}
\sqrt{h} u_{a} g^{i j} £_{\xi} N_{i j}^{a} & =2 \sqrt{h}\left(K_{a b} K^{a b}-u^{a} \nabla_{a} K\right) \\
& =\sqrt{h}\left(g^{\alpha \beta} \partial_{\tau}^{2} g_{\alpha \beta}+\frac{1}{2} \partial_{\tau} g^{\alpha \beta} \partial_{\tau} g_{\alpha \beta}\right)
\end{aligned}
$$

where we have used Eq. (A11). It can be shown that equating this expression to $-16 \pi \bar{T}_{a b} u^{a} u^{b}$ correctly reproduces the standard time-time component of Einstein's equation in the synchronous frame. So, our Eq. (20) gives the correct result, as it should.

As an explicit example, consider the Friedmann universe for which $g_{\alpha \beta}=a^{2}(t) \delta_{\alpha \beta}$ leading to the following expressions:

$$
\partial_{\tau} g_{\alpha \beta}=2 a \dot{a} \delta_{\alpha \beta} ; \quad \partial_{\tau}^{2} g_{\alpha \beta}=\left(2 \dot{a}^{2}+2 a \ddot{a}\right) \delta_{\alpha \beta} ; \quad \partial_{\tau} g^{\alpha \beta}=-2 \frac{\dot{a}}{a^{3}} \delta^{\alpha \beta}
$$

and $\bar{T}_{a b} u^{a} u^{b}=(1 / 2)(\rho+3 p)$. On substitution of Eq. (24), in Eq. (23) we arrive at the following expression for the time evolution of the scale factor:

$$
\frac{\ddot{a}}{a}=-\frac{4 \pi}{3}(\rho+3 p)
$$


The above equation supplemented by the equation of state leads to the standard results. Thus in Friedmann universe the dynamical evolution of spacetime leads to dynamical evolution equation of the scale factor sourced by the Komar energy density. Before proceeding further it is worthwhile to clarify the following point: In the case of Friedmann universe, one can also obtain [35] the following result

$$
\frac{d V}{d t}=N_{\text {sur }}-\sum \epsilon N_{\text {bulk }}
$$

where $V=(4 \pi / 3) H^{-3}$ is the areal volume of the Hubble radius sphere if we define the degrees of freedom using the temperature $T \equiv H / 2 \pi$. (The $\epsilon$ factor has to chosen for each bulk component appropriately in order to keep all $N_{\text {bulk }}$ positive as indicated by the summation; see [35] for a detailed discussion). Though this is also equivalent to Einstein's equation, it is structurally quite different from the evolution equation in Eq. (20) (and should not be confused with it) for the following reasons: (a) The left hand sides of Eq. (20) and Eq. (26) are different. (b) The placement of $\epsilon$-s are different in the right hand sides of Eq. (20) and Eq. (26). (c) One uses Friedmann time coordinate in the left hand side of Eq. (26) but still attributes a temperature $T \equiv H / 2 \pi$ to define the degrees of freedom. (d) Most importantly, Eq. (26) holds only for Friedmann universe while Eq. (20) is completely general.

Coming back to the consequences of Eq. (20), since this result is true for any Friedmann universe, it is also true for the de Sitter spacetime written in synchronous (Friedmann) coordinates. The de Sitter metric, as seen by comoving observers has an explicit time dependence $a(t) \propto \exp (H t)$ and for these observers the perceived Davies-Unruh temperature vanishes. Nevertheless, Eq. (20) will of course give the correct evolution equation. On the other hand, de Sitter spacetime can also be expressed in static coordinates with the line element:

$$
d s^{2}=-\left(1-\frac{r^{2}}{l^{2}}\right) d t^{2}+\frac{d r^{2}}{\left(1-\frac{r^{2}}{l^{2}}\right)}+r^{2}\left(d \theta^{2}+\sin ^{2} \theta d \phi^{2}\right)
$$

The observers with $x^{\alpha}=$ constant in this coordinate system are not geodesic observers. They have the following four velocity and four acceleration respectively:

$$
\begin{aligned}
u_{a} & =\sqrt{\left(1-\frac{r^{2}}{l^{2}}\right)}(-1,0,0,0) \\
a^{i} & =\left(0,-\left(r / l^{2}\right), 0,0\right)
\end{aligned}
$$

Let us see what happens when we use this foliation.

In this case, the acceleration $a^{i}$ and the normal $r_{i}$ are directed opposite to each other as $r_{i}$ is the outward directed normal. (Note that in the de Sitter spacetime the free-falling observers are moving outwards and with respect to them the static observers are moving inwards opposite to the outward pointing normal.) Hence in this situation we have $\epsilon=-1$. The magnitude of the acceleration is:

$$
a=\frac{r}{l^{2}} \frac{1}{\sqrt{\left(1-\frac{r^{2}}{l^{2}}\right)}}
$$

which is obtained from Eq. (29). Thus the local Davies-Unruh temperature turns out to be:

$$
T_{l o c}=\frac{N a}{2 \pi}=\frac{r}{2 \pi l^{2}}=T_{a v g}
$$

Since the spacetime is static $\xi_{i}$ becomes a time-like Killing vector and the Lie derivative of the connection present in Eq. (20) vanishes. Therefore, in this foliation, holographic equipartition should hold. To verify this explicity, we start by calculating surface degrees of freedom. From Eq. (17) the surface degrees of freedom turns out to be:

$$
N_{\text {sur }} \equiv A=\int_{\partial \mathcal{R}} \sqrt{\sigma} d^{2} x=4 \pi r^{2}
$$

Again the bulk degree of freedom can be obtained from Eq. (19) as:

$$
N_{b u l k}=4 \pi \frac{\frac{8 \pi}{3} \rho r^{3}}{r l^{-2}}
$$


Note that the $\epsilon$ factor in the definition of the bulk degrees of freedom, keeps it positive, even though the Komar energy density is negative. Then in de Sitter spacetime we have $8 \pi \rho=\left(3 / l^{2}\right)$ from which we readily observe that:

$$
N_{\text {bulk }}=(8 \pi \rho)\left(l^{2} / 3\right) 4 \pi r^{2}=4 \pi r^{2}=N_{\text {sur }}
$$

Hence for de Sitter spacetime in static coordinates holographic equipartition does hold as it should. (Alternatively, setting $N_{\text {bulk }}=N_{\text {sur }}$ will lead to the correct identification of $l$ in the metric with source by $8 \pi \rho=\left(3 / l^{2}\right)$.)

One can easily verify, by explicit computation, how these results generalize to any static spherically symmetric one, with the line element:

$$
d s^{2}=-f(r) d t^{2}+\frac{d r^{2}}{f(r)}+r^{2} d \Omega^{2}
$$

which covers several interesting metrics with horizons. In this static coordinates the holographic equipartition holds, as can be easily checked. A more interesting situation is in the case of geodesic observers in a synchronous frame. To check this, we start with a coordinate transformation: $(t, r, \theta, \phi) \rightarrow(\tau, R, \theta, \phi)$ in which the variables are related by the following equations:

$$
\begin{aligned}
d t & =d R-\frac{1}{\sqrt{1-f(r)}} \frac{d r}{f(r)} \\
d R & =d \tau+\frac{d r}{\sqrt{1-f(r)}}
\end{aligned}
$$

In terms of these newly defined variables the line element reduces to the synchronous form:

$$
d s^{2}=-d \tau^{2}+[1-f(r)] d R^{2}+r^{2} d \Omega^{2}
$$

The comoving observers, having four velocities $u_{a}=(-1,0,0,0)$ are geodesic observers with zero acceleration and thus the local Davies-Unruh temperature also becomes zero. We can use Eq. (22) and Eq. (23) to describe the evolution. The relevant derivatives are:

$$
\begin{aligned}
\partial_{\tau} g_{R R} & =-f^{\prime}(r) \dot{r} ; \quad \partial_{\tau}^{2} g_{R R}=-f^{\prime}(r) \ddot{r}-f^{\prime \prime}(r) \dot{r}^{2} ; \quad \partial_{\tau} g^{R R}=\frac{f^{\prime}(r) \dot{r}}{[1-f(r)]^{2}} \\
\partial_{\tau} g_{\theta \theta} & =2 r \dot{r} ; \quad \partial_{\tau}^{2} g_{\theta \theta}=2 r \ddot{r}+2 \dot{r}^{2} ; \quad \partial_{\tau} g^{\theta \theta}=-\frac{2 \dot{r}}{r^{3}} \\
\partial_{\tau} g_{\phi \phi} & =2 r \dot{r} \sin ^{2} \theta ; \quad \partial_{\tau}^{2} g_{\theta \theta}=\left(2 r \ddot{r}+2 \dot{r}^{2}\right) \sin ^{2} \theta ; \quad \partial_{\tau} g^{\theta \theta}=-\frac{2 \dot{r}}{r^{3}} \frac{1}{\sin ^{2} \theta}
\end{aligned}
$$

On substitution of these in Eq. (23) we obtain the following differential equation satisfied by the unknown function $f(r)$ :

$$
f^{\prime \prime}(r)+\frac{2 f^{\prime}(r)}{r}=16 \pi \bar{T}_{\tau \tau}=-16 \pi \bar{T}_{0}^{0}
$$

It can be easily verified that this is the correct field equation in this case (see e.g., page 302 of ref. [19]). For example, if we consider the metric of a charged particle with $\bar{T}_{\tau \tau}=Q^{2} / 8 \pi r^{4}$ above equation can be solved to give $f(r)=1-(2 M / r)+\left(Q^{2} / r^{2}\right)$, which, of course, is the Reissner-Nordström metric. The description being covariant but foliation dependent, is actually very desirable and inevitable feature from the thermodynamical point of view $[36,37]$.

\section{GENERALIZATION TO LANCZOS-LOVELOCK GRAVITY}

In the previous section we have reviewed, in the context of Einstein-Hilbert action, how the departure from holographic equipartition leads to the dynamics of the spacetime and have also shown that in static spacetime the surface degrees of freedom equals the bulk degrees of freedom. We will now generalize the above description to Lanczos-Lovelock gravity. 


\section{A. A Brief Introduction to Lanczos-Lovelock Gravity}

Consider, in a $\mathrm{D}$ dimensional spacetime, an action functional which is made from the metric and the curvature tensor but does not contain any derivatives of curvature tensor, such that:

$$
A=\int_{\mathcal{V}} d^{D} x \sqrt{-g} L\left(g^{a b}, R_{b c d}^{a}\right) .
$$

Let us define:

$$
P^{a b c d}=\left(\frac{\partial L}{\partial R_{a b c d}}\right)_{g_{i j}}
$$

which has all the algebraic properties of the curvature tensor. We next define another tensor (which is a generalization of Ricci tensor in general relativity) by

$$
\mathcal{R}^{a b} \equiv P^{a i j k} R^{b}{ }_{i j k} .
$$

This tensor is actually symmetric though the result is nontrivial to prove (for this result and more properties of these tensors see [38]). The variation of the action functional leads to:

$$
\begin{aligned}
\delta A & =\delta \int_{\mathcal{V}} d^{D} x \sqrt{-g} L \\
& =\int_{\mathcal{V}} d^{D} x \sqrt{-g} E_{a b} \delta g^{a b}+\int_{\mathcal{V}} d^{D} x \sqrt{-g} \nabla_{j} \delta v^{j}
\end{aligned}
$$

where we have the following expression for equation of motion term $E_{a b}$ and the boundary term $\delta v^{a}$ :

$$
\begin{aligned}
E_{a b} & \equiv \frac{1}{\sqrt{-g}}\left(\frac{\partial \sqrt{-g} L}{\partial g^{a b}}\right)_{R_{a b c d}}-2 \nabla^{m} \nabla^{n} P_{a m n b} \\
& =\mathcal{R}_{a b}-\frac{1}{2} g_{a b} L-2 \nabla^{m} \nabla^{n} P_{a m n b} \\
\delta v^{j} & =2 P^{i b j d} \nabla_{b} \delta g_{d i}-2 \delta g_{d i} \nabla_{c} P^{i j c d} .
\end{aligned}
$$

This is fairly general but we impose the condition that the field equation should be second order in the metric. Since the quantity $P^{a b c d}$ involves second derivative of the metric, the term $\nabla^{m} \nabla^{n} P_{a m n b}$ in $E_{a b}$ contains fourth order derivative of the metric. We can get second order field equation by imposing an extra condition on $P^{a b c d}$ such that:

$$
\nabla_{a} P^{a b c d}=0
$$

Thus finding an action functional which would lead to equations of motion which are second order in the metric reduces to finding scalars such that Eq. (47) is satisfied. Such an action functional is unique and coincides with Lanczos-Lovelock Lagrangian in D dimensions given by [39-42]:

$$
L=\sum_{m} c_{m} L^{(m)}=\sum_{m} c_{m}\left(\delta_{c d c_{2} d_{2} \ldots c_{m} d_{m}}^{a b a_{2} b_{2} \ldots a_{m} b_{m}} R_{a_{2} b_{2}}^{c_{2} d_{2}} \ldots R_{a_{m} b_{m}}^{c_{m} d_{m}}\right) R_{a b}^{c d}
$$

Due to complete antisymmetry in the indices of the determinant tensor, we have in a D dimensional spacetime the following restriction $2 m \leq D$. (Otherwise the determinant tensor would vanish identically.) In four dimensions, this property uniquely fixes the result to be the Einstein-Hilbert action for $m=1$. The nature of Lanczos-Lovelock models at $D=2 \mathrm{~m}$ is of quiet importance as these are known as critical dimensions for a given Lanczos-Lovelock term. In these situations the variation of action functional reduces to a pure surface term [43].

To proceed further, we need the expression for the Noether current in Lanczos-Lovelock gravity. Recall that the standard result for the Noether current, for diffeomorphism invariance of a Lagrangian $L\left(g^{a b}, R_{b c d}^{a}\right)$, is given by [9]:

$$
16 \pi J^{a}=2 E_{b}^{a} \xi^{b}+L \xi^{a}+\delta_{\xi} v^{a}
$$


where $E_{a b}$ is defined in Eq. (45) and $\delta_{\xi} v^{a}$ represents the surface term in the Lagrangian variation. The following three relations can be used:

$$
\begin{aligned}
2 E_{b}^{a} \xi^{b}+L \xi^{a} & =2 \mathcal{R}_{b}^{a} \xi^{b} \\
\delta_{\xi} v^{a}=-£_{\xi} v^{a} & =-2 \mathcal{R}_{b}^{a} \xi^{b}+2 P^{a b d i} \nabla_{b} \nabla_{d} \xi_{i} \\
\delta_{\xi} v^{i} & =2 P_{a}^{b c i} £_{\xi} \Gamma_{b c}^{a}
\end{aligned}
$$

to express the Noether current in two different, useful, forms as follows:

$$
\begin{aligned}
16 \pi J^{a} & =2 \mathcal{R}_{b}^{a} \xi^{b}+\delta_{\xi} v^{a}=2 P^{a b c d} \nabla_{b} \nabla_{c} \xi_{d} \\
& =2 \mathcal{R}_{b}^{a} \xi^{b}+2 P_{i}{ }^{j k a} £_{\xi} \Gamma_{j k}^{i} .
\end{aligned}
$$

The corresponding expression for the Noether potential in Lanczos-Lovelock gravity is given by [9]:

$$
16 \pi J^{a b}(\xi)=2 P^{a b c d} \nabla_{c} \xi_{d} .
$$

We can obtain the entropy of horizons from the relevant Noether charge. In Lanczos-Lovelock gravity the entropy is defined in terms of the tensor $P^{a b c d}$ and is known as Wald entropy with the expression [44-51]:

$$
S=-\frac{1}{8} \int d^{D-2} x \sqrt{\sigma} P^{a b c d} \mu_{a b} \mu_{c d} \equiv \int d^{D-2} x s
$$

where $\sigma$ is the metric determinant over the $(D-2)$ dimensional hypersurface and $\mu_{a b}$ is the bi-normal to the hypersurface. The last equation defines the entropy density $s$ which will be used frequently in our later discussion.

\section{B. Heat Content of Spacetime in Lanczos-Lovelock Gravity}

We will work with the same spacetime foliations defined in Eq. (2) throughout and thus will use the vectors $u^{a}, \xi^{a}$. We begin by performing the same calculation as before, viz. connecting the Noether charge in a volume to the heat content of the boundary. To do this we will start by relating the Noether current for a vector $q_{a}$ to that of another vector $f(x) q_{a}=v_{a}$ for any arbitrary function $f(x)$. From App. A 2 using Eq. (A16) we obtain the desired relation as:

$$
16 \pi\left\{q_{a} J^{a}(f q)-f q_{a} J^{a}(q)\right\}=\nabla_{b}\left(2 P^{a b c d} q_{a} q_{d} \nabla_{c} f\right)
$$

The usefulness of the above equation again originates from the fact that if $q_{a}=\nabla_{a} \phi$ then its Noether current vanishes and thus Noether current for $v_{a}=f(x) q_{a}$ acquires a particularly simple form. Applying the above result for the two natural vector fields $u^{a}$ and $\xi^{a}$ from Eq. (A24) we obtain the simple relation:

$$
16 \pi u_{a} J^{a}(\xi)=2 D_{\alpha}\left(N \chi^{\alpha}\right)
$$

where we have introduced a new vector field $\chi^{a}$ given by [see Eq. (A18)]:

$$
\chi^{a}=-2 P^{a b c d} u_{b} u_{d} a_{c}
$$

which satisfies the condition $u_{a} \chi^{a}=0$ (so that it is a spatial vector) and also has the property: $D_{i} \chi^{i}=$ $\nabla_{i} \chi^{i}-a_{i} \chi^{i}$. We can integrate Eq. (58) over $(D-1)$ dimensional volume bounded by $N=$ constant surface within $t=$ constant hypersurface leading to:

$$
\int_{\mathcal{V}} d^{D-1} x \sqrt{h} u^{a} J_{a}(\xi)=\int_{\partial \mathcal{V}} \frac{d^{D-2} x \sqrt{\sigma}}{8 \pi} N r_{\alpha} \chi^{\alpha}
$$

As in general relativity, here also the vector $r_{\alpha}$ is the unit normal to the $N=$ constant hypersurface. This vector is either parallel or anti-parallel to the acceleration four vector such that $r_{\alpha}=\epsilon a_{\alpha} / a$, where 
$\epsilon=+1$ implies parallel to acceleration and vice-versa. With this notion, we obtain the following result from the vector field $\chi_{\alpha}$ :

$$
\sqrt{\sigma} \frac{N r_{\alpha} \chi^{\alpha}}{8 \pi}=\epsilon\left(\frac{N a}{2 \pi}\right)\left(\frac{1}{2} \sqrt{\sigma} P^{\alpha b d \beta} r_{\alpha} u_{b} u_{d} r_{\beta}\right)
$$

The term in brackets is closely related to the entropy density of the surface in Lanczos-Lovelock gravity, defined in Eq. (56) as [9, 45]:

$$
s=-\frac{1}{8} \sqrt{\sigma} P^{a b c d} \mu_{a b} \mu_{c d}=\frac{1}{2} \sqrt{\sigma} P^{\alpha b d \beta} r_{\alpha} u_{b} u_{d} r_{\beta} .
$$

Using this expression for entropy density in Eq. (61) we obtain:

$$
\sqrt{\sigma} \frac{N r_{\alpha} \chi^{\alpha}}{8 \pi}=\epsilon T_{l o c} s
$$

where $T_{l o c}=N a / 2 \pi$ is the redshifted local Unruh-Davies temperature as measured by the observers moving normal to $t=$ constant surface, with respect to the local vacuum of freely falling observers. We thus see that the results in general relativity has a natural generalization to Lanczos-Lovelock models. With all these results, Eq. (60) reduces to:

$$
\int_{\mathcal{V}} d^{D-1} x \sqrt{h} u^{a} J_{a}(\xi)=\epsilon \int_{\partial \mathcal{V}} d^{D-2} x T_{l o c} s .
$$

Thus in Lanczos-Lovelock gravity as well the Noether charge in a bulk region is equal to the surface heat content of the boundary. The similar result derived for general relativity can be thought of as a special case of the Lanczos-Lovelock gravity; the connection between the bulk Noether charge and the surface heat content goes way beyond the general relativity. This result is nontrivial because the expression for entropy density in the general Lanczos-Lovelock models is nontrivial in contrast with general relativity in which it is just one quarter per unit area.

\section{Evolution Equation of Spacetime in Lanczos-Lovelock Gravity}

Let us next generalize our result presented in Eq. (1) for Lanczos-Lovelock models obtaining the dynamical evolution as due to deviation from holographic equipartition. We will start by substituting the Noether current expression for $\xi^{a}$ as presented in Eq. (54) to Eq. (58) which leads to the following result:

$$
2 u_{a} P_{i}^{j k a} £_{\xi} \Gamma_{j k}^{i}=D_{\alpha}\left(2 N \chi^{\alpha}\right)-2 N \mathcal{R}_{a b} u^{a} u^{b}
$$

Let us first consider the pure Lanczos-Lovelock theory with the $m$ th order Lanczos-Lovelock Lagrangian. (We shall consider the generalization to Lanczos-Lovelock models with a sum of Lagrangians, at the end.) Contracting the field equation $\mathcal{R}_{a b}-(1 / 2) g_{a b} L=8 \pi T_{a b}$ in Lanczos-Lovelock gravity with $g^{a b}$ we get $L=[8 \pi] /[m-(D / 2)] T$, where $D$ is space-time dimension. Therefore field equation can also be rewritten as:

$$
\mathcal{R}_{a b}=8 \pi \bar{T}_{a b}=8 \pi\left(T_{a b}-\frac{1}{2} \frac{1}{(D / 2)-m} g_{a b} T\right) \equiv 8 \pi \bar{T}_{a b}
$$

Using this and integrating Eq. (65) over $(D-1)$ dimensional volume we arrive at:

$$
\int_{\mathcal{R}} \frac{d^{D-1} x \sqrt{h}}{8 \pi} 2 u_{a} P_{i}{ }^{j k a} £_{\xi} \Gamma_{j k}^{i}=\int_{\partial \mathcal{R}} \frac{d^{D-2} x \sqrt{\sigma}}{4 \pi} N \chi^{\alpha} r_{\alpha}-\int_{\mathcal{R}} d^{D-1} x \sqrt{h} 2 N \bar{T}_{a b} u^{a} u^{b} .
$$

As before, the $r_{\alpha}$ is the normal to $N=$ constant surface within $t=$ constant surface and is either parallel or anti-parallel to the four acceleration. The energy momentum term can be written in an identical 
fashion by using the Komar energy density, defined as: $\rho_{\text {Komar }}=2 N \bar{T}_{a b} u^{a} u^{b}$. We can proceed using Eq. (61), which on substitution into Eq. (67) leads to:

$$
\int_{\mathcal{R}} \frac{d^{D-1} x \sqrt{h}}{8 \pi} 2 u_{a} P_{i}^{j k a} £_{\xi} \Gamma_{j k}^{i}=-2 \epsilon \int_{\partial \mathcal{R}} d^{D-2} x \sqrt{\sigma} P^{\alpha b \beta d} r_{\alpha} u_{b} r_{\beta} u_{d}\left(\frac{1}{2} T_{l o c}\right)-\int_{\mathcal{R}} d^{D-1} x \sqrt{h} \rho_{K \text { Komar }}
$$

Rest of the analysis requires proper definition of $N_{s u r}, N_{b u l k}$ etc which we do in analogy with the case of general relativity. The number of surface degrees of freedom is defined as four times the entropy as in the case of general relativity:

$$
N_{\text {sur }} \equiv 4 S=2 \int_{\partial \mathcal{R}} d^{D-2} x \sqrt{\sigma} P^{\alpha b d \beta} r_{\alpha} u_{b} u_{d} r_{\beta}
$$

The average temperature is properly defined using the surface degrees of freedom as the local weights leading to ensure that the total heat content is reproduced:

$$
\frac{1}{2} N_{\text {sur }} k_{B} T_{a v g}=\frac{1}{2} \int d N_{\text {sur }} k_{B} T_{l o c} ; \quad T_{a v g} S=\int T_{l o c} d S
$$

This result can be written, more explicitly as:

$$
T_{a v g}=\frac{\int_{\partial \mathcal{R}} d^{D-2} x \sqrt{\sigma} P^{\alpha b \beta d} r_{\alpha} u_{b} r_{\beta} u_{d} T_{l o c}}{\int_{\partial \mathcal{R}} d^{D-2} x \sqrt{\sigma} P^{\alpha b \beta d} r_{\alpha} u_{b} r_{\beta} u_{d}}=\frac{1}{S} \int d S T_{l o c}=\frac{1}{N_{\text {sur }}} \int d N_{\text {sur }} T_{l o c}
$$

Once $T_{a v g}$ is defined, the number of bulk degrees of freedom is given by the equipartition value:

$$
N_{\text {bulk }}=\frac{\epsilon}{(1 / 2) T_{\text {avg }}} \int_{\mathcal{R}} d^{D-1} x \sqrt{h} \rho_{\text {Komar }}
$$

with $\epsilon$ included (as in general relativity), to ensure that $N_{\text {bulk }}$ is always positive. Inserting Eqs. (69), (71) and (72) in Eq. (68) we find that the dynamical evolution of the spacetime in Lanczos-Lovelock gravity is determined by the following relation:

$$
\int_{\mathcal{R}} \frac{d^{D-1} x \sqrt{h}}{8 \pi} 2 u_{a} P_{i}^{j k a} £_{\xi} \Gamma_{j k}^{i}=\epsilon\left(\frac{1}{2} T_{a v g}\right)\left(N_{\text {sur }}-N_{\text {bulk }}\right)
$$

which is direct generalization of the corresponding result for general relativity.

For a static spacetime the Lie variation of connection vanishes as $\xi^{a}$ becomes a time-like Killing vector. Hence in that situation we have, even in in Lanczos-Lovelock gravity, the holographic equipartition given by:

$$
N_{s u r}=N_{b u l k}
$$

(This result has been obtained earlier in terms of equipartition energies in ref. [52].) When the foliation leads to time dependent metric, the departure from holographic equipartition drives dynamical evolution of the metric through the Lie derivative term on the left hand side of Eq. (73).

The above result was derived for $m$ th order Lanczos-Lovelock Lagrangian. The definition of $\bar{T}_{a b}, \rho_{K o m a r}$ and $N_{\text {bulk }}$ introduces the $m$ dependence though the expression for $\mathcal{R}_{a b}$ in Eq. (66). If, instead, we consider a Lanczos-Lovelock Lagrangian made of a sum of Langrangians with different $m$, then the equation of motion, $\mathcal{R}_{a b}-(1 / 2) g_{a b} L=8 \pi T_{a b}$ on contraction with $g^{a b}$ leads to the result:

$$
\sum_{m} c_{m}[m-(D / 2)] L_{(m)}=8 \pi T
$$

which cannot be solved in closed form for $L$ in terms of $T$. However, one can take care of this issue by redefining $\rho_{\text {Komar }}$ and $N_{\text {bulk }}$ formally in terms of $\mathcal{R}_{a b}$. That is, we define the Komar energy density as: $\rho=2 N\left(\mathcal{R}_{a b} / 8 \pi\right) u^{a} u^{b}$ and then the bulk degrees of freedom reduces to the following form:

$$
N_{\text {bulk }}=\frac{\epsilon}{(1 / 2) T_{\text {avg }}} \int_{\mathcal{R}} d^{D-1} x \sqrt{h} \rho
$$


Then we again obtain the same result:

$$
\int_{\mathcal{R}} \frac{d^{D-1} x \sqrt{h}}{8 \pi} 2 u_{a} P_{i}{ }^{j k a} £_{\xi} \Gamma_{j k}^{i}=\epsilon\left(\frac{1}{2} T_{\text {avg }}\right)\left(N_{\text {sur }}-N_{\text {bulk }}\right)
$$

with the understanding that, for a given model, one should re-express the variables in terms of $T_{a b}$.

The above results provide a direct connection between evolution of spacetime and departure from holographic equipartition. The results also encode the holographic behavior of gravity by introducing naturally defined bulk and surface degrees of freedom. The difference between the description of evolution along these lines and that of standard field equation $\mathcal{R}_{a b}-(1 / 2) g_{a b} L=8 \pi T_{a b}$ is the following: For the standard gravitational field equations the left hand side does not have a clear physical meaning. There is also no distinction between static and dynamic spacetime and hence the standard treatment cannot answer the question: what drives the time-dependence of the metric? The answer is obviously not $T_{a b}$ since we can obtain time dependent solutions even when $T_{a b}=0$ and static solutions with $T_{a b} \neq 0$. In contrast the evolution depicted in Eq. (77) addresses all these issues and we have a natural separation between static and evolving metrics via holographic equipartition. When the surface and bulk degrees of freedom are unequal, resulting in departure from holographic equipartition, it drives the time-dependence of the metric. Thus the driving force behind dynamical evolution of spacetime is the departure from holographic equipartition, providing a physically transparent statement about spacetime dynamics.

\section{DISCUSSION}

Our aim in this work was to consider the relationship between the Noether current and gravitational dynamics in a useful manner. Noether currents can be thought of as originating from mathematical identities in differential geometry, with no connection to the diffeomorphism invariance of gravitational action [31]. This result holds not only in general relativity but also in Lanczos-Lovelock gravity (see Appendix A 1 ).

Even though such conserved currents can be associated with any vector field, the time development vectors are always special. This is the motivation for introducing the vector $\xi^{a}$ in the spacetime through Eq. (2). The vector $\xi^{a}$ is parallel to velocity vector $u^{a}$ for fundamental observers and represents proper time flow normal to $t=$ constant surface. As we saw, its Noether charge and current associated with this vector have elegant and physically interesting thermodynamic interpretation. We showed that, for the vector field $\xi^{a}$ in Lanczos-Lovelock gravity in arbitrary spacetime dimension, total Noether charge in any bulk volume $\mathcal{V}$, bounded by constant lapse surface, equals the heat content of the boundary surface. Also the equipartition energy of the surface equals twice the Noether charge. While defining the heat content, we have used local Unruh-Davies temperature and Wald entropy. This result holds for Lanczos-Lovelock gravity of all orders and does not rely on static spacetime or existence of Killing vector like criteria.

The above identification allow us to study holographic equipartition for static spacetime and relate the time evolution of the metric as due to departure from holographic equipartition. With a suitable and natural definition for the degrees of freedom in the surface and in the bulk, we find that for static spacetimes (described in the natural foliation) the surface and the bulk degrees of freedom are equal in number yielding holographic equipartition. It is the departure from this holographic equipartition that drives spacetime evolution. This result holds not only in general relativity but also in Lanczos-Lovelock gravity.

All the results derived above are generally covariant but they do depend on the foliation. This implies that these results depend on observers and their acceleration which is inevitable since the Davies-Unruh temperature is intrinsically observer dependent. Since the dynamical evolution is connected to thermodynamic concepts in this approach, different observers must perceive the dynamical evolution differently. For example, the de Sitter spacetime is time dependent when written in synchronous frame, becomes time independent in static spherically symmetric coordinate. Our description adapts naturally to the two different situations. 


\section{Acknowledgement}

Research of T.P is partially supported by J.C. Bose research grant of DST, Govt. of India. Research of S.C is funded by SPM Fellowship from CSIR, Govt. of India. S.C also likes to thank Krishnamohan Parattu, Suprit Singh and Kinjalk Lochan for helpful discussions. We thank Naresh Dadhich for useful comments.

\section{Appendix A: Calculational Details}

Some calculations are not presented in an explicit format in the main text, which would affect the flow of ideas in the paper. Most of these relations exist in the literature; however we collect the derivations together here with the hope that they will be useful to the reader.

\section{Derivation of Noether Current from differential Identities in Lanczos-Lovelock Gravity}

In this section the Noether current for Lanczos-Lovelock gravity will be derived starting from identities in differential geometry without using any difeomorphism invariance of action principles. The conceptual importance of this approach has already been emphasized in ref. [31], in the context of Einstein gravity, and we shall generalize the result for Lanczos-Lovelock models. We start with the fact that the covariant derivative of any vector field can be decomposed into a symmetric and an antisymmetric part. From the antisymmetric part we can define another antisymmetric tensor field as,

$$
16 \pi J^{a j}=2 P^{a j k i} \nabla_{k} v_{i}=P^{a j k i}\left(\nabla_{k} v_{i}-\nabla_{i} v_{k}\right)
$$

It is evident from the antisymmetry of $P^{a b c d}$ that a conserved current exists such that, $J^{a}=\nabla_{j} J^{a j}$. We recall the identities:

$$
\left(\nabla_{j} \nabla_{k}-\nabla_{k} \nabla_{j}\right) v^{i}=R_{c j k}^{i} v^{c}
$$

and,

$$
\mathcal{L}_{v} \Gamma_{j k}^{i}=\nabla_{j} \nabla_{k} v^{i}-R_{k j m}^{i} v^{m}
$$

and use them in the definition in Eq. (43) to get:

$$
\begin{aligned}
\mathcal{R}^{a b} v_{b} & =P^{a i j k} R_{i j k}^{b} v_{b}=-P^{a i j k}\left(\nabla_{j} \nabla_{k}-\nabla_{k} \nabla_{j}\right) v_{i} \\
& =P^{a i j k} \nabla_{k} \nabla_{j} v_{i}+\left(P^{a k i j}+P^{a j k i}\right) \nabla_{j} \nabla_{k} v_{i} \\
& =P^{a i j k} \nabla_{k} \nabla_{j} v_{i}+P^{a k i j} \nabla_{j} \nabla_{k} v_{i}+\nabla_{j}\left(P^{a j k i} \nabla_{k} v_{i}\right)
\end{aligned}
$$

where in the second line we have used the identity, $P^{a(b c d)}=0$. Then from Eq. (A1) we obtain:

$$
\begin{aligned}
16 \pi J^{a} & =2 \mathcal{R}^{a b} v_{b}-2 P^{a i j k} \nabla_{k} \nabla_{j} v_{i}-2 P^{a k i j} \nabla_{j} \nabla_{k} v_{i} \\
& =2 \mathcal{R}^{a b} v_{b}+2 P_{i}{ }^{a j k} \nabla_{k} \nabla_{j} v^{i}-2 P_{i}{ }^{j a k} \nabla_{j} \nabla_{k} v^{i} \\
& =2 \mathcal{R}^{a b} v_{b}+2 P_{i}{ }^{a j k}\left(\mathcal{L}_{v} \Gamma_{k j}^{i}+R^{i}{ }_{j k m} v^{m}\right)-2 P_{i}{ }^{j a k}\left(\mathcal{L}_{v} \Gamma_{j k}^{i}+R_{k j m}^{i} v^{m}\right) \\
& =2 \mathcal{R}^{a b} v_{b}+2 P_{i}{ }^{j k a} \mathcal{L}_{v} \Gamma_{j k}^{i}
\end{aligned}
$$

while arriving at the third line we have used Eq. (A3) and for the last line we have used the fact that, $P^{i j a k} R_{i k j m}=P^{a k i j} R_{i k j m}=-P^{k a i j} R_{i k j m}=P^{k a i j} R_{k i j m}$. Thus Eq. (54) can be derived without any reference to the diffeomorphism invariance of the gravitational action, using only the identities in differential geometry and various symmetry properties. 


\section{Identities Regarding Noether current in Lanczos-Lovelock Action}

The Noether potential $J^{a b}$ is antisymmetric in $(a, b)$ and from its expression given by Eq. (6) it is evident that $J^{a b}(q)$ would identically vanish for $q_{a}=\nabla_{a} \phi$. We will use the above fact in order to obtain a relation between the Noether current for two vector fields $q_{a}$ and $v_{a}$ connected by $v_{a}=f(x) q_{a}$. This result, in the case of general relativity is detailed in ref. [31]. Expanding the expression for Noether current for $v_{a}=f q_{a}$ and taking dot product with $q_{a}$ along with subtracting the Noether current for $q_{a}$ one can show that:

$$
16 \pi\left\{q_{a} J^{a}(f q)-f q_{a} J^{a}(q)\right\}=\nabla_{b}\left[\left(q^{a} q^{b}-q^{2} g^{a b}\right) \nabla_{a} f\right] .
$$

This is the result used in the main text. Using this result it is easy to detemine the Noether currents for $u_{a}=-N \nabla_{a} t$ and $\xi_{a}=N u_{a}$. Using Eq. (A6) with $q_{a}=-u_{a} / N$ and $f=-N$ we obtain:

$$
16 \pi u_{a} J^{a}(u)=\nabla_{i} a^{i}-a^{2}=D_{\alpha} a^{\alpha}
$$

where the acceleration is defined as:

$$
a_{j}=u^{i} \nabla_{i} u_{j}=\left(u^{i} \nabla_{i} N\right) \frac{u_{j}}{N}+N u^{i} \nabla_{j}\left(\frac{u_{i}}{N}\right)=h_{j}^{i} \frac{\nabla_{i} N}{N} .
$$

Next in order to obtain the Noether current for $\xi^{a}$ we use Eq. (A6) with $q_{a}=u_{a}$ and $f=N$ leading to:

$$
16 \pi u_{a} J^{a}(\xi)=N u_{a} J^{a}(u)+\nabla_{j}\left(N a^{j}\right)=2 N \nabla_{j} a^{j}=D_{\alpha}\left(2 N a^{\alpha}\right)
$$

which is the desired relation in Eq. (8).

In general relativity the quantity $u_{a} g^{i j} £_{\xi} N_{i j}^{a}$ can be evaluated in terms of the extrinsic curvature [19]. Then from the standard identity

$$
\nabla_{i} a^{i}-R_{a b} u^{a} u^{b}=K_{i j} K^{i j}-u^{a} \nabla_{a} K
$$

we obtain:

$$
u_{a} g^{i j} £_{\xi} N_{i j}^{a}=2 N\left(\nabla_{i} a^{i}-R_{a b} u^{a} u^{b}\right)=2 N\left(K_{i j} K^{i j}-u^{a} \nabla_{a} K\right)
$$

Next we will generalize the above results to Lanczos-Lovelock gravity. For that purpose we note that even in Lanczos-Lovelock gravity the Noether potential $J^{a b}$ for a vector field $q_{a}=\nabla_{a} f$ vanishes identically. Thus the Noether current for a vector field $v_{a}=f(x) q_{a}$ can be decomposed as:

$$
\begin{aligned}
16 \pi J^{a b}(v) & =2 P^{a b c d} \nabla_{c}\left(f q_{d}\right) \\
& =2 P^{a b c d} q_{d} \nabla_{c} f+2 f P^{a b c d} \nabla_{c} q_{d}
\end{aligned}
$$

Then the corresponding Noether current has the following expression:

$$
\begin{aligned}
16 \pi J^{a}(v) & =2 P^{a b c d} \nabla_{b}\left(q_{d} \nabla_{c} f\right)+2 P^{a b c d} \nabla_{b}\left(f \nabla_{c} q_{d}\right) \\
& =2 P^{a b c d} q_{d} \nabla_{b} \nabla_{c} f+2 P^{a b c d} \nabla_{c} f \nabla_{b} q_{d}+2 P^{a b c d} \nabla_{b} f \nabla_{c} q_{d}+2 f P^{a b c d} \nabla_{b} \nabla_{c} q_{d}
\end{aligned}
$$

From the above equation we readily arrive at:

$$
\begin{aligned}
16 \pi\left\{J^{a}(v)-f J^{a}(q)\right\} & =2 P^{a b c d} q_{d} \nabla_{b} \nabla_{c} f+2 P^{a b c d} \nabla_{c} f \nabla_{b} q_{d}+2 P^{a b c d} \nabla_{b} f \nabla_{c} q_{d} \\
& =P^{a b c d} \nabla_{b} A_{c d}+16 \pi J^{a b}(q) \nabla_{b} f
\end{aligned}
$$

where we have defined the antisymmetric tensor $A_{c d}$ as $A_{c d}=q_{d} \nabla_{c} f-q_{c} \nabla_{d} f$. Now consider the following result: $q_{a} \nabla_{b} A_{c d}=\nabla_{b}\left(q_{a} A_{c d}\right)-A_{c d} \nabla_{b} q_{a}$ which leads to:

$$
\begin{aligned}
P^{a b c d} q_{a} \nabla_{b} A_{c d} & =\nabla_{b}\left(P^{a b c d} q_{a} A_{c d}\right)-2 P^{a b c d} q_{d} \nabla_{c} f \nabla_{b} q_{a} \\
& =\nabla_{b}\left(P^{a b c d} q_{a} A_{c d}\right)-16 \pi q_{a} J^{a b}(q) \nabla_{b} f
\end{aligned}
$$

Then Eq. (A14) can be rewritten in the following manner:

$$
\begin{aligned}
16 \pi\left\{q_{a} J^{a}(f q)-f q_{a} J^{a}(q)\right\} & =16 \pi J^{a b}(q) \nabla_{b} f q_{a}+\nabla_{b}\left(P^{a b c d} q_{a} A_{c d}\right)-16 \pi q_{a} J^{a b}(q) \nabla_{b} f \\
& =\nabla_{b}\left(2 P^{a b c d} q_{a} q_{d} \nabla_{c} f\right)
\end{aligned}
$$


It can be easily verified that in the general relativity limit $P^{a b c d}=Q^{a b c d}=(1 / 2)\left(g^{a c} g^{b d}-g^{a d} g^{b c}\right)$, under which the above equation reduces to Eq. (A6).

Applying the above equation to $u_{a}=-N \nabla_{a} t$ with $q_{a}=\nabla_{a} t=-u_{a} / N$ and $f=-N$ we arrive at:

$$
16 \pi u_{a} J^{a}(u)=2 N \nabla_{b}\left(P^{a b c d} u_{a} u_{d} \frac{\nabla_{c} N}{N^{2}}\right)
$$

In order to proceed we define a new vector field such that:

$$
\begin{aligned}
\chi^{a} & =-2 P^{a b c d} u_{b} u_{d} \frac{\nabla_{c} N}{N} \\
& =-2 P^{a b c d} u_{b} u_{d}\left(a_{c}-\frac{1}{N} u_{c} u^{j} \nabla_{j} N\right) \\
& =-2 P^{a b c d} u_{b} a_{c} u_{d}
\end{aligned}
$$

Note that in the general relativity limit this vector reduces to the acceleration four vector as follows:

$$
\chi^{a}=-2 P^{a b c d} u_{b} a_{c} u_{d}=-\left(g^{a c} g^{b d}-g^{a d} g^{b c}\right) u_{b} a_{c} u_{d}=-u^{b} u_{b} a^{a}+u^{b} a_{b} u^{a}=a^{a}
$$

Also just as in the case of acceleration for the vector $\chi^{a}$ as well we have:

$$
u_{a} \chi^{a}=-2 a P^{a b \beta d} u_{a} u_{b} r_{\beta} u_{d}=0
$$

where antisymmetry of $P^{a b c d}$ in the first two components has been used. We can also have the following relation for the vector field $\chi^{a}$ :

$$
N a_{b} \chi^{b}=\chi^{b} \nabla_{b} N+\chi^{b} u_{b} u^{j} \nabla_{j} N=\chi^{b} \nabla_{b} N
$$

where we have used the relation $u_{a} \chi^{a}=0$ from Eq. (A20). Thus Eq. (A17) can be written in terms of the newly defined vector field $\chi^{a}$ in the following way:

$$
\begin{aligned}
16 \pi u_{a} J^{a}(u) & =N \nabla_{b}\left(\frac{\chi^{b}}{N}\right) \\
& =\nabla_{b} \chi^{b}-\frac{\nabla_{b} N}{N} \chi^{b} \\
& =D_{\alpha} \chi^{\alpha}
\end{aligned}
$$

The last relation follows from the fact that:

$$
D_{\alpha} \chi^{\alpha}=D_{b} \chi^{b}=\nabla_{b} \chi^{b}-a_{b} \chi^{b}=\nabla_{b} \chi^{b}-\frac{\nabla_{b} N}{N} \chi^{b}
$$

Then it is straightforward to get the Noether current for $\xi^{a}$ by using $q_{a}=u_{a}$ and $f=N$ in Eq. (A16) with Eq. (A22) as:

$$
\begin{aligned}
16 \pi u^{a} J_{a}(\xi) & =16 \pi N u_{a} J^{a}(u)+\nabla_{b}\left(N \chi^{b}\right) \\
& =N D_{\alpha} \chi^{\alpha}+\nabla_{b}\left(N \chi^{b}\right) \\
& =D_{\alpha}\left(2 N \chi^{\alpha}\right)
\end{aligned}
$$

Here also we have used the following identity:

$$
\begin{aligned}
D_{\alpha}\left(N \chi^{\alpha}\right) & =\left(g^{i j}+u^{i} u^{j}\right) \nabla_{i}\left(N \chi_{j}\right) \\
& =\nabla_{i}\left(N \chi^{i}\right)+u^{i} u^{j} \nabla_{i}\left(N \chi_{j}\right) \\
& =N \nabla_{i} \chi^{i}+N \chi^{i} a_{i}-N \chi^{j}\left(u^{i} \nabla_{i} u_{j}\right) \\
& =N \nabla_{i} \chi^{i}
\end{aligned}
$$

Thus we have derived the desired relation for the Noether current of the vector field $\xi_{a}$ and it turns out to have identical structure as that of general relativity action with $\chi^{a}$ playing the role of four acceleration. 
[1] J.D. Bekenstein, Phys. Rev. D 7, 2333 (1973).

[2] J.D. Bekenstein, Phys. Rev. D 9, 3292 (1974).

[3] S. Hawking, Commun. Math. Phys. 43, 199 (1975).

[4] S. Hawking, Phys. Rev. D 13, 191 (1976).

[5] P. C. W. Davies, S. A. Fulling, and W. G. Unruh, Phys. Rev. D 13, 2720 (1976).

[6] W. G. Unruh, Phys. Rev. D 14, 870 (1976).

[7] T. Padmanabhan, Phys. Rept. 406, 49 (2005) [arXiv:0311036].

[8] R.M. Wald, Liv. Rev. Relt. 4, 6 (2001) [arXiv:9912119].

[9] T. Padmanabhan, Rept. Prog. Phys. 73, 046901 (2010) [arXiv:0911.5004].

[10] A.D. Sakharov, Sov. Phys. Dokl. 12, 1040 (1968).

[11] T. Jacobson, Phys. Rev. Lett. 75, 1260 (1995).

[12] G.E. Volovik, Phys. Rept. 351, 195 (2001).

[13] T. Padmanabhan, Class. Quant. Grav. 19, 5387 (2002) [arXiv:0204019].

[14] R.G. Cai and S.P. Kim, J. High Energy Phys. 0502, 050 (2005) [arXiv:hep-th/0501055].

[15] A. Paranjape, S. Sarkar and T. Padmanabhan, Phys. Rev. D 74, 104015 (2006) [arXiv:hep-th/0607240].

[16] M. Akbar and R.G. Cai, Phys. Lett. B 635, 7 (2006) [arXiv:hep-th/0602156].

[17] T. Padmanabhan, AIP Conference Proceedings 861, 858 (2006) [arXiv:astro-ph/0603114].

[18] D. Kothawala and T. Padmanabhan, Phys. Rev. D 79, 104020 (2009) [arXiv:0904.0215].

[19] T. Padmanabhan, Gravitation: Foundation and Frontiers, Cambridge University Press, Cambridge, UK (2010).

[20] A. Mukhopadhyay and T. Padmanabhan, Phys. Rev. D 74, 124023 (2006) [arXiv:hep-th/0608120].

[21] S. Kolekar and T. Padmanabhan, Phys. Rev. D 82, 024036 (2010) [arXiv:1005.0619].

[22] S. Kolekar, D. Kothawala and T. Padmanabhan, Phys. Rev. D 85, 064031 (2012) [arXiv:1111.0973].

[23] T. Padmanabhan, Braz. J. Phys. 35, 362 (2005) [arXiv:0412068].

[24] T. Padmanabhan, Mod. Phys. Lett. A25, 1129 (2010) [arXiv:0912.3165].

[25] G. Gibbons and S.W. Hawking, Phys. Rev. D 15, 2752 (1977).

[26] T. Padmanabhan and A. Paranjape, Phys. Rev. D 75, 064004 (2007) [arXiv:0701003].

[27] T. Padmanabhan, Gen. Relt. Grav. 40, 529 (2008) [arXiv:0705.2533].

[28] T. Padmanabhan, Phys. Rev. D 83, 044048 (2011) [arXiv:1012.0119].

[29] S. Kolekar and T. Padmanabhan, Phys. Rev. D 85, 024004 (2012) [arXiv:1109.5353].

[30] T. Damour, Surface Effects in Black Hole Physics, Proceedings of the second Marcel Grossmann Meeting on General Relativity (1982).

[31] T. Padmanabhan, Gen. Rel. Grav 46, 1673 (2014) [arXiv:1312.3253].

[32] S. Babak and L. Grishchuk, Phys. Rev. D 61, 024038 (1999) [arXiv:9907027].

[33] J. Kijowski, Gen. Relt. Grav. 29, 307 (1997).

[34] K. Parattu, B.R. Majhi and T. Padmanabhan, Phys. Rev. D 87, 124011 (2013) [arXiv:1303.1535].

[35] T. Padmanabhan, Res. Astro. Astrophys., 12, 891 (2012) [arXiv:1207.0505]

[36] T. Padmanabhan, AIP Conf. Proc. 1483, 212 (2012) [arXiv:1208.1375].

[37] T. Padmanabhan, AIP Conf. Proc. 1241, 93 (2010) [arXiv:0911.1403].

[38] T. Padmanabhan, Phys. Rev. D 84, 124041 (2011) [arXiv:1109.3846].

[39] C. Lanczos, Z.Phys. 73, 147 (1932); C. Lanczos, Ann. of Math. 39, 842 (1938).

[40] D. Lovelock, J. Math. Phys. 12, 498 (1971).

[41] T. Padmanabhan and D. Kothawala, Phys. Rept. 531, 115 (2013).

[42] A. Eddington, The Mathematical Theory of Relativity, Cambridge University Press, Cambridge, UK (1924); E. Schrodinger, Space-time Structure, Cambridge University Press, Cambridge, UK (1950).

[43] A. Yale and T. Padmanabhan, Gen. Relt. Grav. 43, 1549 (2011) [arXiv:1008.5154]; N. Kiriushcheva and S. Kuzmin, Mod. Phys. Lett. A21 (2006) [arXiv:hep-th/0510260].

[44] R.M. Wald, Phys. Rev. D 48, R3427 (1993);

V. Iyer and R.M. Wald, Phys. Rev. D 50, 846 (1994);

R.M. Wald and A. Zoupas, Phys. Rev. D 61, 084027 (2000).

[45] T. Padmanabhan, Gen. Relt. Grav. 44, 2681 (2012) [arXiv:1205.5683].

[46] B.R. Majhi and T. Padmanabhan, Eur. Phys. J C 73, 2651 (2013) [arXiv:1302.1206].

[47] B.R. Majhi and T. Padmanabhan, Phys. Rev. D 85, 084040 (2012) [arXiv:1111.1809].

[48] B.R. Majhi, Adv. High Energy Phys. 386342 (2013) [arXiv:1210.6736].

[49] A. Strominger and C. Vafa, Phys. Lett. B 379, 99 (1996).

[50] A. Ashtekar, J. Baez, A. Corichi and K. Krasnov, Phys. Rev. Lett. 80, 904 (1998); J.M. Garcia-Islas, Class. Quant. Grav. 25, 245001 (2008).

[51] L. Bombelli, R.K. Koul, J. Lee and R.D. Sorkin, Phys. Rev. D 34, 373 (1986). 
[52] T. Padmanabhan, Phys. Rev. D 81, 124040 (2010) [arXiv:1003.5665]. 